\title{
A prospective, single-centre, randomised study evaluating the clinical, imaging and immunological depth of remission achieved by very early versus delayed Etanercept in patients with Rheumatoid Arthritis (VEDERA)
}

Raluca B. Dumitru ${ }^{1,2}$, Sarah Horton 1,2, Richard Hodgson ${ }^{3}$, Richard J. Wakefield ${ }^{1,2}$, Elizabeth M. A. Hensor ${ }^{1,2}$, Paul Emery ${ }^{1,2}$ and Maya H. Buch ${ }^{1,2^{*}}$

\begin{abstract}
Background: Rheumatoid arthritis (RA) is a chronic inflammatory arthritis, with significant impact on quality of life and functional status. Whilst biologic disease modifying anti-rheumatic drugs (bDMARD) such as tumour necrosis factor-inhibitor (TNFi) agents have revolutionised outcomes in RA, early diagnosis with immediate conventional therapy, titrated in a treat to target approach is also associated with high remission rates. The main aim of the VEDERA study (Very Early versus Delayed Etanercept in Rheumatoid Arthritis) is to assess the depth of remission, sustainability of remission and immunological normalisation induced by very early TNFi with etanercept (ETN) or standard of care +/- delayed ETN.
\end{abstract}

Methods/Design: VEDERA is a pragmatic, phase IV single-centre open-label randomised superiority trial of 120 patients with early, treatment-naive RA. Patients will be randomised 1:1 to first-line ETN and methotrexate (MTX) or MTX with additional synthetic disease modifying anti-rheumatic drugs (sDMARDs) according to a treat to target (TT) protocol with further step up to ETN and MTX after 24 weeks if remission is not achieved. Participants will have regular disease activity assessments and imaging evaluation including musculoskeletal ultrasound and MRI. The main objective of this study is to assess the proportion of patients with early RA that achieve clinical remission at 48 weeks, following either treatment strategy. In addition, the participants are invited to take part in a cardio-vascular sub-study (Coronary Artery Disease in RA, CADERA), which aims to identify the incidence of cardiovascular abnormalities in early RA.

Discussion: The hypothesis underlining this study is that very early treatment with first-line ETN increases the proportion of patients with rheumatoid arthritis achieving clinical remission, in comparison to conventional therapy.

Trial registration: NCT02433184, 23/04/2015

Keywords: Rheumatoid arthritis, Disease modifying anti-rheumatic drug, Etanercept, Treat to target, Remission, Clinical efficacy, MRI synovitis score, Molecular predictors of response

\footnotetext{
* Correspondence: m.buch@leeds.ac.uk

${ }^{1}$ Leeds Institute of Rheumatic and Musculoskeletal Medicine, University of Leeds, Leeds, UK

${ }^{2} \mathrm{NIHR}$ Leeds Musculoskeletal Biomedical Research Unit, Leeds Teaching

Hospitals NHS Trust, Leeds, UK

Full list of author information is available at the end of the article
} 


\section{Background}

Rheumatoid arthritis (RA) is a chronic inflammatory arthritis, characterised by symmetrical, often erosive, inflammatory polyarthritis of the small and medium sized joints, which can lead to significant disability and decreased function [1]. The overall burden of disease however extends beyond the joints to include wide-ranging extra-articular manifestations and comorbidities [2].

The fundamental paradigm in the management of RA is that early, effective suppression of synovitis by early diagnosis and immediate initiation of disease modifying anti rheumatic drug (DMARD) therapy is associated with improved outcomes. Guidelines recommend synthetic DMARD (sDMARD) as a first line therapy with step-up to biological DMARD (bDMARD) treatment in the presence of continued disease activity. Internationally agreed guidelines recommend a treat to target (TT) approach, a concept established for the management of pathologies such as arterial hypertension and diabetes [3]. In RA, TT implies frequent monitoring using a composite disease activity measure such as the disease activity score-28 joint (DAS28) and escalation of therapy according to a pre-defined target of remission or at least low disease activity (LDA) [4, 5]. Tight control of disease activity with intensive treatment, either step-up [6] or parallel $[7,8]$ in early RA is associated with an improvement in medium and long term outcomes.

The introduction of bDMARDs has provided highly effective therapy with which to achieve effective disease control [9-11]. There are currently five licensed tumournecrosis factor-inhibitor (TNFi) therapies, the first bDMARDs to be approved for use, of which etanercept (ETN) is a recombinant TNF receptor fused to a human Fc molecule forming a bivalent TNF binding agent. ETN reduces inflammation, improves functional status and quality of life and inhibits radiographic progression $[12,13]$. As with other TNFi, ETN is recommended following MTX-inadequate response [14], and in the UK after failure of at least two sDMARDs (of which one should be MTX) [15].

Recent studies including COMET [16], ASPIRE [17] and OPTIMA [18], suggest that TNFi therapy in the early phase of the disease, prior to failure of sDMARDs, offers improved rates of response and inhibition of radiographic progression in comparison to SDMARD. Sub-analysis of the COMET study shows that patients receiving very early treatment with ETN and methotrexate (within the first 4 months of disease) achieve greater rates of remission than those with longer disease [19]. Furthermore, studies suggest that when commenced early, TNFi may offer a greater depth of remission that is more likely to be sustained after drug withdrawal [20]. In recent years, increasing consideration has been given to the 'window of opportunity' concept: a phase in early disease in which it may be possible to alter the pathogenic course of the disease [21]. However, not all studies demonstrate the superiority of TNFi when compared to $\mathrm{TT}$ regimen in early disease; in a group of DMARD-naive early RA patients, no statistically significant differences were found between patients receiving MTX and intravenous steroids, followed by TT regimen and combination therapy with MTX and infliximab [22]. There is thus, continued debate on how best to apply TNFi therapies in order to obtain the maximal benefits to the patient whilst considering socio- and health-economic costs. In addition, increasing research is aimed at identifying predictors of response. This includes use of TNFi within a TT strategy [17, 18], and for remission [23].

An additional challenge is the assessment of therapeutic response. Whilst clinical scores such as DAS28 form the basis of evaluation, it is accepted that these may be insensitive [24]; imaging modalities such as power Doppler (PD) ultrasound (US) and dynamic magnetic resonance imaging (MRI) enable more accurate assessment of inflammation as well as providing insights into the pathobiology of disease [25].

\section{Methods/Design}

\section{Aims and objectives}

The main aim of the VEDERA study (Very Early versus Delayed ETN in Rheumatoid Arthritis) is to evaluate the depth of remission, sustainability of remission and immunological normalisation induced by very early TNFi or the current standard of care (with or without delayed TNFi if required), in patients with early, treatment-naive RA.

\section{Research hypothesis}

Very early treatment with first-line ETN increases the proportion of patients with rheumatoid arthritis achieving clinical remission, in comparison to conventional therapy.

\section{Primary objective}

The primary objective of this study is to assess the proportion of patients with early, treatment-naive RA that achieve clinical remission at 48 weeks following first-line ETN and MTX ('very early ETN') or MTX with additional sDMARDs and step up to ETN and MTX at 24 weeks, as required according to a TT protocol aiming for remission ('delayed ETN').

\section{Secondary objectives}

- To assess the change in MRI synovitis between baseline and 48 weeks following very early ETN or MTX-TT regimen +/- delayed ETN. 
- To evaluate other clinical efficacy endpoints at weeks 12, 24, 48 and 96 including:

$\checkmark$ Disease activity score-44 joint (DAS44) remission (DAS44 < 1.6), simplified disease activity index (SDAI), clinical disease activity index (CDAI), European League Against Rheumatism (EULAR) response criteria, American College of Rheumatology (ACR) response criteria.

$\checkmark$ Cumulative steroid dose.

- To evaluate patient-reported outcomes at weeks 12 , 24, 48 and 96 including:

$\checkmark$ Physical function, assessed by the Health Assessment Questionnaire (HAQ), including normalisation of HAQ.

$\checkmark$ Quality of life scores assessed by RA Quality of Life (RA-QoL) and Euro QoL Five Dimensions (EQ-5D) questionnaires.

$\checkmark$ Work instability, assessed by RA Work Instability Score (RA-WIS) questionnaire.

- To evaluate imaging predictors of remission and sustained remission using:

$\checkmark$ High-resolution ultrasound (HRUS) at weeks $0,12,24$ and 48 measuring grey scale (GS), $\mathrm{PD}$ and presence or absence of erosions.

$\checkmark$ Magnetic Resonance Imaging (MRI) at weeks $0,12,24$ and 96 measuring synovitis and erosion scores.

- To evaluate other radiographic outcomes including:

$\checkmark$ Joint damage assessed by modified Sharp score of X-rays of hands and feet at weeks 48 and 96.

\section{Exploratory objectives}

- To investigate immunological abnormalities and cellular/molecular predictors of response to the two therapeutic strategies using:

$\checkmark$ Blood, sampled at baseline and weeks 12, 24, 36, 48 and 96.

$\checkmark$ Synovial tissue (optional), biopsied from a target joint at baseline and 24 weeks; at 48 weeks for treatment arm 2 who have switched to ETN and at time of flare.

\section{Study design}

The VEDERA study is a phase IV single-centre open-label randomised trial of 120 patients with early, treatmentnaive RA. Patients will be randomised 1:1 to first-line ETN and MTX or MTX with additional sDMARDs and step up to ETN and MTX after 24 weeks as required according to a TT protocol aiming for remission.

The randomised trial duration is 48 weeks after which ETN will be stopped and patients will continue for a 48-week observational period during which
DMARDs will be used as per standard care, in accordance with national guidelines.

\section{Patient eligibility}

The target population are males and females, aged between 18 and 80 years, fulfilling the 2010 American College of Rheumatology/European League against Rheumatism (ACR/EULAR) RA classification criteria, who have not yet received DMARD therapy, with a maximum symptom duration of 12 months. Other criteria for inclusion are: patients with active RA at baseline (clinical evidence of synovitis and DAS28ESR > 3.2) and positive anti-citrullinated peptide antibody (ACPA) or rheumatoid factor (RF) status. In the absence of RF and ACPA antibodies, patients are eligible for the study if they have evidence of power Doppler, a good indicator of active inflammatory pathology and predictor of structural damage. Power Doppler grade $\geq 1$ in at least 1 joint on hand ultrasound is required (Table 1).

\section{Recruitment}

Participants will be recruited from the rheumatology clinics based within Leeds Teaching Hospitals NHS Trust. The recruitment period is expected to last up to 36 months. All patients in the VEDERA study will be invited to participate in CADERA, the cardiovascular sub-study.

\section{Screening}

Following written informed consent and prior to any trial related procedures, participants will be registered and undergo a screening assessment that should occur no more than four weeks prior to the baseline assessments, to determine eligibility for the study.

\section{Randomisation}

Once eligibility is confirmed and all relevant procedures have been completed, participants will be block randomised 1:1 to one of the following two treatment groups (Fig. 1):

- Treatment Arm 1 or 'very early ETN' will receive ETN and MTX combination therapy administered for a total duration of 48 weeks.

- Treatment Arm 2 or 'delayed ETN' will receive initial MTX monotherapy with adoption of a TT protocol (standard care involving monthly DAS28ESR assessment with escalation to combination sDMARD therapy if not achieving LDA at, or after, 8 weeks) and step-up to ETN and MTX at 24 weeks if failing to achieve clinical remission. 
Table 1 Eligibility criteria for randomisation into VEDERA trial

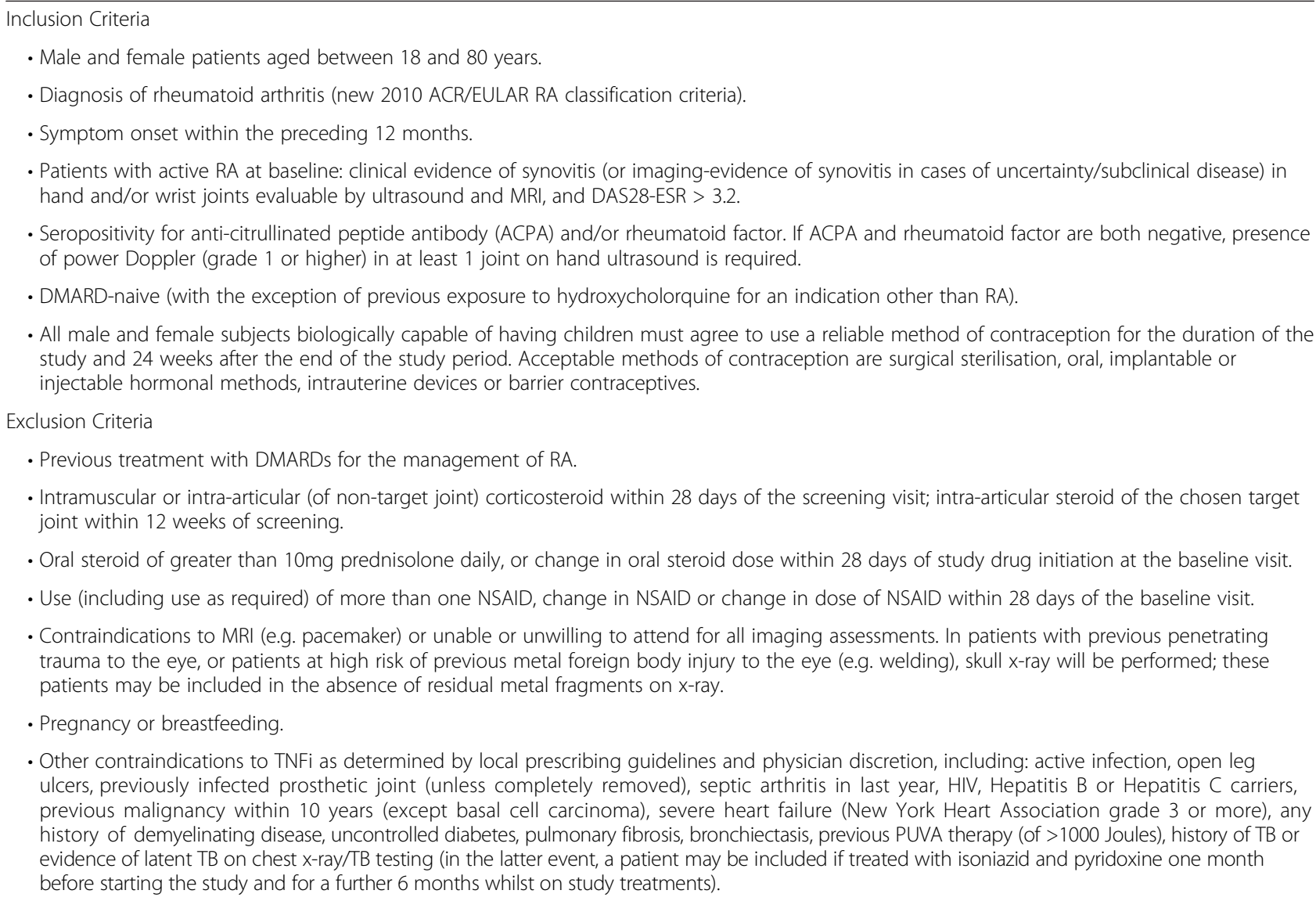

\section{Trial intervention}

\section{Treatment arm one}

For treatment arm one (Table 2), ETN will be administered subcutaneously up to week 48 (except in the case of intolerance or subject withdrawal) at a dose of $50 \mathrm{mg}$ weekly. MTX will be administered orally at a starting dose of $15 \mathrm{mg}$ weekly, increasing to $20 \mathrm{mg}$ and $25 \mathrm{mg}$ weekly at weeks 4 and 8 respectively. Patients that are clear non-responders or intolerant to ETN and/or MTX will be managed according to physician discretion (withdrawing these study treatments if appropriate).

\section{Treatment arm two}

In treatment arm two (Table 2), MTX will be administered orally at a starting dose of $15 \mathrm{mg}$ and will be increased to $25 \mathrm{mg}$ weekly at 2 weeks. Subcutaneous MTX may be administered if intolerance to oral MTX is observed. If at weeks $8,12,16$ or 20 , the subject fails to achieve LDA (defined as DAS28-ESR $\leq 3.2$ ), sulfasalazine (SSZ) and hydroxychloroquine (HCQ) will be added to MTX. SSZ will be administered orally at a dose of $1 \mathrm{~g}$ twice daily (or at the maximum tolerated dose). HCQ will be administered at a dose of $200 \mathrm{mg}$ daily. At 24 weeks, if a subject fails to achieve clinical remission,
ETN will be added to MTX, and SSZ and HCQ will be discontinued (if applicable).

\section{Treatment arms 1 and 2}

Oral folic acid will be administered at a dose of $5 \mathrm{mg}$ daily (except on the day of MTX) to subjects in both treatment arms.

ETN will be discontinued in both arms at the primary endpoint (48 weeks), with the exception of those patients who are eligible to continue according to local prescribing guidelines (NICE guidelines) [15].

A single non-steroidal anti-inflammatory drug (NSAID) is permitted providing the dose has been unchanged for at least 28 days prior to study drug initiation at baseline. The NSAID or NSAID dose may be changed during the time course of the study if indicated.

Oral prednisolone is permitted at doses up to and including $10 \mathrm{mg}$ prednisolone daily if the dose has been stable for at least 28 days prior to study drug initiation at baseline. The steroid dose may be reduced throughout the study.

Intramuscular steroid may be administered as per the study treatment protocol for each arm with all patients 


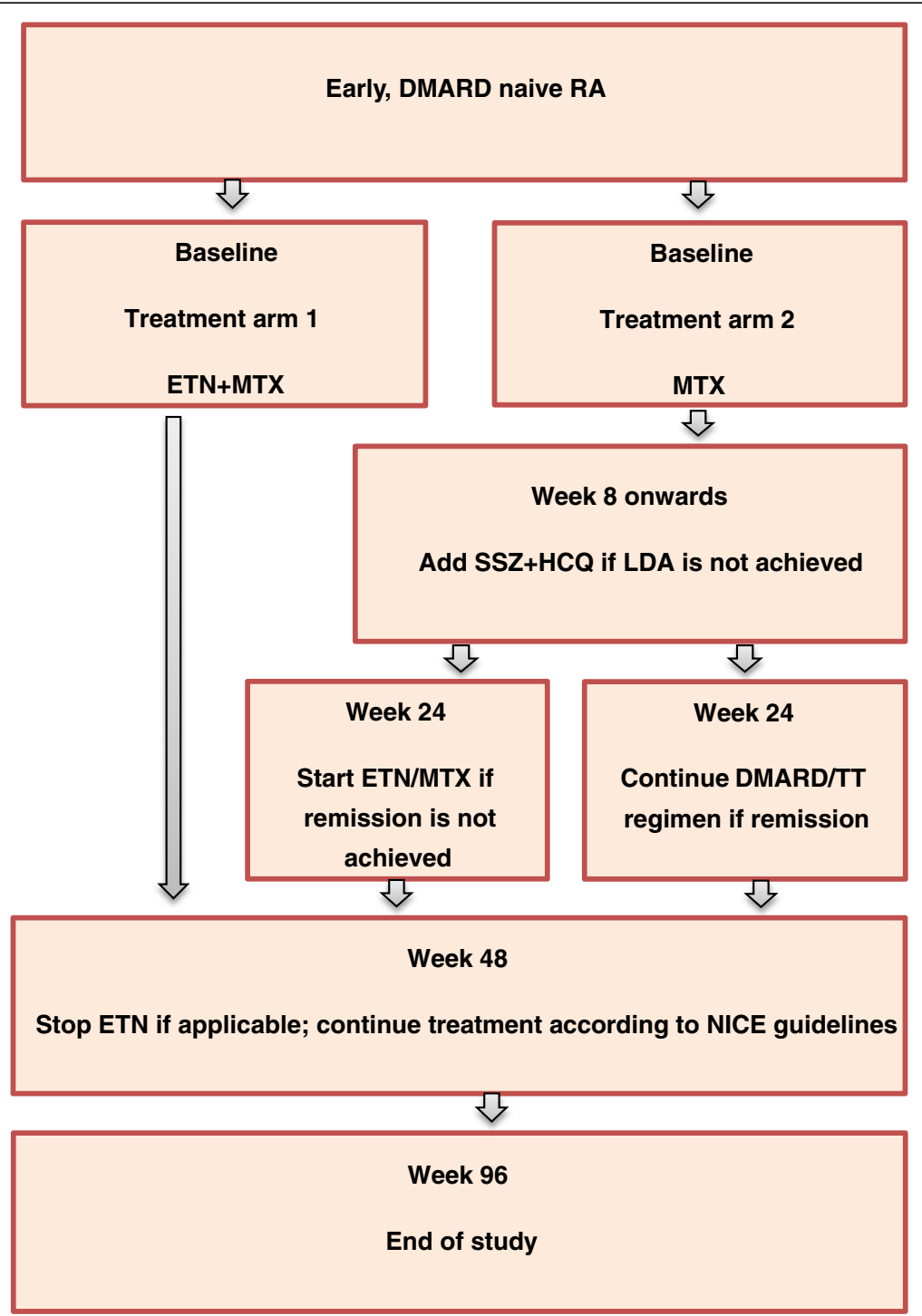

Fig. 1 Schematic of the trial design. DMARD disease modifying antirheumatic drugs, ETN etanercept, HCQ hydroxychloroquine, LDA low disease activity, MTX methotrexate, NICE National Institute of Clinical Excellence, RA rheumatoid arthritis, SSZ sulfasalazine, TT treat to target

receiving 120mg intramuscular methylprednisolone (unless contraindicated or not tolerated) at baseline and at the following time-points according to treatment arm and disease activity:

- Week 12: both treatment arms, if DAS28-ESR > 3.2

- Week 24: treatment arm 1, if DAS28-ESR $\geq 2.6$ (treatment arm 2 switch to ETN+/-MTX if DAS28-ESR $\geq 2.6$ )

- Week 36:

$\checkmark$ Treatment arm 1, if DAS28-ESR $\geq 2.6$

$\checkmark$ Treatment arm 2, sDMARDs (MTX +/-SSZ $+/$-HCQ), if DAS28-ESR $\geq 2.6$

$\checkmark$ Treatment arm 2, switched to ETN+/-MTX, if DAS28-ESR $>3.2$
- Up to the primary endpoint (week 48), for a patient with active disease who is judged by the physician to be in need of rescue therapy, and for whom it is considered to be unethical to wait until the 12, 24 and 36 week time points above, $120 \mathrm{mg}$ methylprednisolone may be administered.

At and/or after the primary endpoint (week 48) intramuscular or intra-articular steroids are permitted according to physician judgment, unless a clinical or imaging assessment is scheduled within the following 6 weeks.

Alternative DMARDs, other than study treatments, are permitted if clinically indicated in a subject judged by the physician to be a non-responder (primary or secondary non-responder) or intolerant to ETN. 
Table 2 Treatment arm scheme

\begin{tabular}{|c|c|c|c|}
\hline Arm 1 & Dosage form & Dosage regimen & Duration \\
\hline ETN & Subcutaneous & 50mg weekly & Up to week 48 unless non-response or intolerance. \\
\hline MTX & $\begin{array}{l}2.5 / 10 \mathrm{mg} \text { tablets } \\
\text { (or s.c.if oral not tolerated) }\end{array}$ & $\begin{array}{l}\text { Weeks 0-4: } 15 \mathrm{mg} \text { weeklyWeek 4: } 20 \mathrm{mg} \\
\text { weeklyWeek } 8 \text { onwards: } 25 \mathrm{mg} \text { weekly }\end{array}$ & $\begin{array}{l}\text { For duration of study (unlessintolerance: aim for } \\
\text { maximumtolerated dose) }\end{array}$ \\
\hline Folic Acid & $5 \mathrm{mg}$ tablets & 5mg, six days per week (not day of MTX) & For duration of study (if receiving MTX) \\
\hline \multicolumn{4}{|l|}{ Arm 2} \\
\hline MTX & $\begin{array}{l}2.5 / 10 \mathrm{mg} \text { tablets } \\
\text { (or s.c. if oral not tolerated) }\end{array}$ & $\begin{array}{l}\text { Weeks 0-2: } 15 \text { mg weeklyWeek } 2 \text { onwards: } \\
25 \text { mg weekly }\end{array}$ & $\begin{array}{l}\text { For duration of study(unless intolerance, } \\
\text { aim formaximum tolerated dose) }\end{array}$ \\
\hline Folic Acid & $5 \mathrm{mg}$ tablets & $5 \mathrm{mg}$ six days per week (not day of MTX) & For duration of study (if receiving MTX) \\
\hline SSZ & $500 \mathrm{mg}$ tablets & $1 \mathrm{~g}$ twice a day & $\begin{array}{l}\text { At/after week } 8 \text { if not achieving low disease activity } \\
\text { (DAS28ESR }>3.2 \text {. Discontinuation if ETN is started } \\
\text { at week 24. }\end{array}$ \\
\hline HCQ & $200 \mathrm{mg}$ tablets & $200 \mathrm{mg}$ tablets & $\begin{array}{l}\text { At/after week } 8 \text { if not achieving low disease activity } \\
\text { (DAS28ESR }>3.2 \text {. Discontinuation if ETN is started } \\
\text { at week 24. }\end{array}$ \\
\hline ETN & Subcutaneous & 50mg weekly & $\begin{array}{l}\text { At week } 24 \text { to week } 48 \text { if not achieving remission } \\
\text { (DAS28ESR } \geq 2.6) \text { Thereafter continuation will be } \\
\text { determined by physician judgement/according } \\
\text { to national guidelines. }\end{array}$ \\
\hline
\end{tabular}

ETN Etanercept, MTX Methotrexate, SSZ Sulfasalazine, HCQ Hydroxychloroquine

\section{Prohibited medications}

Steroids are prohibited prior to week 48, with the exception of:

- Patients receiving $\leq 10 \mathrm{mg}$ prednisolone daily with a stable dose for at least 28 days prior to study drug initiation at baseline.

- Patients receiving prednisolone for an indication other than arthritis, for example asthma or chronic obstructive pulmonary disease (up to a maximum total oral dose of $250 \mathrm{mg}$, and a maximum duration of 14 days).

- Intramuscular steroid as per the study treatment protocol for each arm.

- 120mg methylprednisolone administered as rescue therapy (see above).

In the unavoidable instance of a subject receiving corticosteroid within a significant time window prior to a clinical +/- imaging joint assessment the assessment will not be performed. A significant time window is defined as follows: intramuscular corticosteroid within 4 weeks or oral prednisolone within 7 days of the assessment, except patients on stable prednisolone $\leq 10 \mathrm{mg}$.

At or after week 48, intramuscular or intra-articular steroids are prohibited within 6 weeks of a scheduled clinical or imaging assessment.

Other prohibited medications are any alkylating agents (e.g. cyclophosphamide, chlorambucil), any experimental drugs and immunisations with live vaccines.

\section{Study schedule}

- Screening visit. All patients will undergo screening within the 4 weeks prior to the baseline visit (see section 'Screening' above).

- Baseline visit (week 0). This visit will be performed to confirm eligibility for the study and for randomisation and initiation of study treatment.

- Assessment and treatment visits. These visits will be performed at weeks 4, 12 and every 12 weeks thereafter, up to week 96, for both treatment arms.

- Additional visits (for treatment arm 2). The patients in treatment arm 2 will also be monitored at week 8, 16 and 20 for safety and efficacy within a TT protocol. In patients switching to ETN and MTX at week 24 a follow up safety visit will be arranged 4 weeks following ETN commencement (i.e. week 28).

\section{Methods of assessment Clinical efficacy}

Clinical efficacy and remission will be assessed by the following measures, with joint examinations performed by the same blinded assessor.

DAS44 and DAS28, based on the evaluation of four variables: tender joint assessment (Ritchie Articular Index, RAI, and 28 joint count respectively), number of swollen joints (out of 44 and 28, respectively), erythrocyte sedimentation rate (ESR; $\mathrm{mm} / \mathrm{h}$ ) and patient's global assessment of arthritis, as assessed by a $100 \mathrm{~mm}$ visual analogue scale (VAS). These will be performed at each 
visit for both treatment arms. The scores will be calculated using the following formulae: DAS28 $=0.56$ * sqrt (tender28) $+0.28 *$ sqrt(swollen28) $+0.70 * \ln ($ ESR $)+$ $0.014 *$ VAS; DAS $44=0.54 *$ sqrt $(\mathrm{RAI})+0.065 *$ (swollen $44)+0.33 * \ln ($ ESR $)+0.0072 *$ VAS. Remission thresholds for DAS44 and DAS28 are $<1.6$ and $<2.6$ respectively [26].

$C D A I$ is the sum of four variables: number of tender and swollen joints (out of 28), the investigator's global assessment of disease activity and the patient's global assessment of arthritis assessed by VAS (cm). The threshold for CDAI remission is $<2.8$ [27].

SDAI is the CDAI plus CRP $(\mathrm{mg} / \mathrm{dL})$. The threshold for SDAI remission is $<3.3$ [28].

EULAR response criteria classify patients as good, moderate or non-responders based on a combination of the actual DAS and change from previous DAS [29].

ACR response measures $20 \%$ (ACR20), 50 \% (ACR50) or $70 \%$ (ACR70) improvement in tender and swollen joint counts and in at least three of the following parameters: patient global assessment, physician global assessment, pain, disability and acute phase reactant (ESR or CRP) [30].

The physician global assessment of rheumatoid disease activity should be completed before the patient's global assessment is received. The investigator will mark their assessment on a $100 \mathrm{~mm}$ visual analogue scale (VAS): the left end corresponds to none (0) and the right end to extremely active (100).

\section{Patient-reported outcomes}

Patient general health assessment and pain visual analogue scales (VAS): the patient is instructed to mark their 'general state of health' and 'level of pain' on two $100 \mathrm{~mm}$ scales. The left end (0) corresponds to 'very well' and 'no pain' respectively, and the right end (100) to 'extremely poor' and 'worst possible pain' (100).

The HAQ assesses a patient's level of functional ability. There are 20 questions in 8 categories of functioning that represent different activities - dressing, rising, eating, walking, hygiene, reach, grip and usual activities. For each item there is a 4-level difficulty scale scored from $0-3$, representing no difficulty (0), some (1) or much (2) difficulty, and unable to do (3). The highest component score in each category determines the category score, unless the patient uses aids or devices for, or receives assistance with activities in that category, in which case the relevant category score is increased to 2 if the maximum score was previously $<2$. The 8 category scores are averaged into an overall score from zero to 3 [31].

The RAQoL consists of 30 statements derived directly from relevant patients, using, as far as possible, their own words. Respondents are required to indicate whether or not each of the statements applies to them; each affirmed statement contributes a score of 1 to the total score. Scores can range from 0 to 30 with a [32].

The EQ5D is a generic measure, which provides a single index value [33]. The scale includes a descriptive system, comprising 5 questions relating to different aspects of health each with 3 possible responses ('no problems', 'some problems' and 'extreme problems'), and a visual analogue scale capturing the patient's self-rated health where the endpoints are labelled 'best imaginable health state' and 'worst imaginable health state'.

The RA-WIS [34] consists of 23 statements about the impact of the disease on working. Respondents are required to indicate whether or not each of the statements applies to them; each affirmed statement contributes with a score of 1 to the total score. Scores can range from 0 to 23; a score less than 10 indicate low work instability; scores in the range of 10-17 indicate moderate work instability; scores above 17 indicate high risk of work instability.

Duration of early morning stiffness; the patient is asked to estimate the time that elapsed between awakening and the time he/she is as flexible as he/she will be during a day involving typical activities. Duration in minutes is recorded up to a maximum of $720 \mathrm{~min}(12 \mathrm{~h})$.

\section{Imaging assessments}

MRI (high field gadolinium enhanced) of the dominant hand and wrist (or alternative hand in the instance of greater clinical evidence of synovitis at baseline) will be performed at baseline, weeks 12, 24, 48 and 96. The dominant hand will be scanned unless the alternative hand has clinical evidence of greater synovitis at baseline. This study will use MRI to assess erosions, synovitis and bone marrow oedema using the accepted standard of RA magnetic resonance imaging score (RAMRIS), as well as direct synovial volume measurement and sensitive dynamic contrast enhanced magnetic resonance imaging (DCE-MRI) for quantification of synovitis and bone marrow oedema. Failure of MRI acquisition after baseline will not constitute a protocol violation and will not necessitate withdrawal from the study.

RAMRIS scoring system: scoring of erosions, synovitis and bone marrow oedema, using the validated outcome measures in RA clinical trials (OMERACT) system, will be performed by 2 independent, experienced scorers. The RAMRIS scoring system is a standardised, reliable, validated scoring system for synovitis, erosion and oedema. It has been successfully used to demonstrate response to treatment, including bDMARD [35]. Direct volume measurement of synovitis by manual segmentation will be performed. This will allow quantification of low volumes of synovitis not differentiated by RAMRIS scoring. 
This will be followed by dynamic contrast enhanced (DCE)-MRI. Early enhancement rates measured from DCE-MRI depend strongly on synovial vascularity and capillary permeability and are therefore potentially better markers of inflammatory activity than simple volume measurements. DCE-MRI measurement of bone marrow oedema will also be performed.

A blinded assessor will perform HRUS (Philips HDI 5000) of the same hand and wrist as chosen for MRI at baseline, weeks 12,24 and 48 . If the target joint for synovial biopsy is at another site, the target joint will also be scanned. Images will be assessed for synovitis, using semi-quantitative scores of GS and PD and for presence or absence of erosions.

Plain radiographs of bilateral hands (carpal, MCP and PIP joints) will be performed at baseline, 12 and 24 months after the start of study medication to assess structural damage. Radiographs will be scored as per the modified Genant-modified Sharp scoring system [36].

\section{Exploratory biosample based research}

Blood samples will be collected at baseline and weeks $12,24,36,48,96$, at time of early discontinuation (if indicated) and at time of flare (if indicated). This will include collection of serum, plasma and heparinised blood (for flow cytometry and functional studies).

Synovial biopsy (optional) will be taken from a target joint: an active joint (identified at baseline by presence of PD activity on ultrasound) or alternatively, if necessary, a clinically uninvolved, accessible joint may be chosen. The optional biopsy will be acquired via arthroscopy or under ultrasound guidance at baseline and week 24; at week 48 in patients randomised to treatment arm 2 receiving delayed ETN from week 24, and at time of flare in the case of loss of response in an initial responder. Failure of biopsy acquisition at any time point will not constitute a protocol violation and will not necessitate withdrawal from the study.

The sample collection will be used for wide-ranging studies including cellular phenotyping, soluble biomarker evaluation and gene expression studies.

A detailed study schedule is provided in Table 3.

\section{Follow-up}

At study completion (96 weeks), patients will be invited to take part in the Inflammatory Arthritis Continuum (IACON) study. This is an in-house, prospective, longitudinal, observational study of patients with inflammatory arthritis, with ethical approval to obtain blood samples and clinical and imaging data in consenting patients. Inclusion of all patients with inflammatory arthritis in IACON will allow recording of longer-term outcomes such as sustained response and drug-free remission permitting future observational work.

\section{Statistical considerations Sample size}

Clinical remission (DAS28 remission) forms the basis of the primary outcome. The COMET study, reported remission rates in patients with very early RA (less than 4 months since diagnosis) treated with both ETN and MTX or MTX monotherapy of $70 \%$ versus $35 \%$ respectively. In patients with disease duration of less than 2 years but greater than 4 months, treatment with ETN and MTX was associated with $48 \%$ remission rate. In the COMET study patients in the very early RA group were recruited within 4 months of RA diagnosis, using ACR 1987 criteria. In contrast, VEDERA will recruit patients within 12 months of symptom onset. Whilst all patients will meet RA criteria at the point of recruitment, ACR/EULAR 2010 criteria are being used, which identify patients at an earlier stage of the disease course than the 1987 criteria, and patients can potentially be recruited concurrently with diagnosis. It is likely that patients recruited to VEDERA will be at a similar stage in their disease course to the patients in the very early RA group in COMET, potentially an even earlier stage.

To account for the possibility that the interval between the treatment groups may not be as large as that seen in COMET a $30 \%$ difference has been used as a basis for the sample size calculation. Assuming therefore that patients in treatment arm 1 are likely to achieve up to $70 \%$ remission, and treatment arm 2 approximately $40 \%$ remission; for $80 \%$ power at the $5 \%$ significance level and allowing for a $10 \%$ dropout rate, at least 49 patients per arm will be required. Increasing this to 60 per arm would allow for a subgroup analysis of treatment arm 2 patients that proceed to require delayed ETN and MTX to be compared with treatment arm 1 and indeed those in arm 2 that stay on conventional therapy. This is an exploratory analysis; we estimate that $50 \%$ of treatment arm 2 patients will require delayed ETN and MTX. With roughly 30 patients in each subgroup of treatment arm 2 we will obtain sufficiently accurate estimates of remission rates.

\section{Analysis populations}

The full analysis set will comprise all patients, allocated to treatment groups as originally randomised, irrespective of the treatment received and any subsequent deviations from the study protocol. The per protocol set will comprise patients with primary endpoint data available, whose treatment complies with the study protocol and for whom no other major protocol violations are identified. Protocol violations will be identified during a data review by the study management team, blind to allocation, and prior to locking the study database. 
Table 3 Study schedule

\begin{tabular}{|c|c|c|c|c|c|c|c|c|c|c|c|c|c|c|c|}
\hline & & & & $\operatorname{Arm} 2^{a}$ & & $\operatorname{Arm} 2^{a}$ & $\operatorname{Arm} 2^{a}$ & & & & & & & & \\
\hline Study Week & $\begin{array}{l}\text { Weeks-4 } \\
\text { to } 0\end{array}$ & Week 0 & Week 4 & Week 8 & Week 12 & Week 16 & Week 20 & Week 24 & Week 36 & Week 48 & Week 60 & Week 72 & Week 84 & Week 96 & $\begin{array}{l}\text { Early } \\
\text { discontinuation }\end{array}$ \\
\hline Study Phase & Screen & Baseline & $\begin{array}{l}\text { Safety } \\
\text { visit }\end{array}$ & & & & & & & $\begin{array}{l}1^{0} \\
\text { Endpoint }\end{array}$ & & & & $\begin{array}{l}\text { End of } \\
\text { Study }\end{array}$ & \\
\hline Visit No. & 1 & 2 & 3 & 4 & 5 & 6 & 7 & 8 & 9 & 10 & 11 & 12 & 13 & 14 & \\
\hline $\begin{array}{l}\text { Informed Consent (patient } \\
\text { information will be provided } \\
>24 \text { hrs prior to screen) }\end{array}$ & $x$ & & & & & & & & & & & & & & \\
\hline Inclusion/exclusion criteria & $x$ & $x$ & & & & & & & & & & & & & \\
\hline Randomisation & & $x$ & & & & & & & & & & & & & \\
\hline $\begin{array}{l}\text { Demographics, RA } \mathrm{Hx} \text {, past } \\
\text { medical } \mathrm{Hx} \text {, Family } \mathrm{Hx}, \mathrm{CV} \\
\text { risk factors }{ }^{\mathrm{b}}\end{array}$ & $x$ & & & & & & & & & & & & & & \\
\hline Physical examination ${ }^{c}$ & $x$ & & & & $x$ & & & & & & & & & & $x$ \\
\hline Vital signs $^{d}$ & $x$ & $x$ & $x$ & & $x$ & & & $x$ & $x$ & $x$ & $x$ & $x$ & $x$ & $x$ & $x$ \\
\hline Pregnancy test ${ }^{\mathrm{e}}$ & $x$ & $x$ & & & & & & & & & & & & & \\
\hline ESR, CRP and HS-CRP & $x$ & $x$ & $\begin{array}{l}\text { X (Arm } 2 \\
\text { only) }\end{array}$ & $x$ & $x$ & $x$ & $x$ & $x$ & $x$ & $x$ & $x$ & $x$ & $x$ & $x$ & $x$ \\
\hline $\begin{array}{l}\text { Blood chemistry, } \\
\text { haematology }\end{array}$ & $x$ & $x$ & $x$ & $x$ & $x$ & $x$ & $x$ & $x$ & $x$ & $x$ & $x$ & $x$ & $x$ & $x$ & $x$ \\
\hline Urinalysis & $x$ & $x$ & $x$ & & $x$ & & & $x$ & & $x$ & & $x$ & & $x$ & $x$ \\
\hline Serology: RF, ACPA and ANA & $x$ & & & & & & & & & & & & & $x$ & $x$ \\
\hline ECG & $x$ & & & & & & & & & & & & & & \\
\hline Chest $\mathrm{x}$-ray $/ \mathrm{TB}$ test $\mathrm{f}^{\mathrm{f}}$ & $x$ & & & & & & & & & & & & & & \\
\hline Hepatitis (B and C) serology & $x$ & & & & & & & & & & & & & & \\
\hline $\begin{array}{l}\text { VAS assessments: patient } \\
\text { general health, patient global, } \\
\text { patient pain }\end{array}$ & $x$ & $x$ & $\begin{array}{l}\text { X (Arm } 2 \\
\text { only) }\end{array}$ & $x$ & $x$ & $x$ & $x$ & $x$ & $x$ & $x$ & $x$ & $x$ & $x$ & $x$ & $x$ \\
\hline $\begin{array}{l}\text { Physician VAS global disease } \\
\text { activity }\end{array}$ & $x$ & $x$ & & & $x$ & & & $x$ & & $x$ & & $x$ & & $x$ & $x$ \\
\hline $\mathrm{DAS}_{28 / 44}$ joint assessment ${ }^{9}$ & $x$ & $x$ & $\begin{array}{l}X \text { (Arm } 2 \\
\text { only) }\end{array}$ & $x$ & $x$ & $x$ & $x$ & $x$ & $x$ & $x$ & $x$ & $x$ & $x$ & $x$ & $x$ \\
\hline $\begin{array}{l}\text { Early morning stiffness, } \\
\text { HAQ, RA QoL, EQ-5D, RA-WIS }\end{array}$ & & $x$ & & & $x$ & & & $x$ & & $x$ & & $x$ & & $x$ & $x$ \\
\hline
\end{tabular}

a. Visits at weeks 8, 16 and 20 are only applicable to patients in treatment arm 2. b. Cardiovascular risk factors include: smoking habit (pack years), alcohol intake (units/week), amount of exercise taken, dietary intake (days/week $\geq 5$ portions fruit or vegetables consumed), and family history of premature cardiovascular disease age $<55$ years. c. Physical examination Includes height and body weight at screening. d. Blood pressure after a 5 -min rest, pulse rate and body temperature e. Urinary pregnancy test for women of child bearing potential only $\mathrm{f}$. Chest $\mathrm{x}$-ray, if not already performed within 24 weeks prior to the study. TB testing includes TB Gold Quantiferon +/- T spot test +/- Mantoux test. $\mathrm{g}$. Whenever possible, joint assessments should be performed by the same blinded assessor throughout the time course of the study to reduce potential investigator bias 


\section{Analyses}

Descriptive summary statistics will be provided for all variables at baseline and at each visit at which the variable is assessed. For continuous variables the following information will be provided: number of patients $(\mathrm{N})$, mean, standard deviation, minimum, median, maximum and $25 \%$ and $75 \%$ quartiles. For categorical data frequency (absolute and relative) distributions will be provided. Differences between groups will be summarized according to data type and $95 \%$ confidence intervals around these estimates will be constructed.

All inferential analyses will be conducted using twotailed tests at the $5 \%$ level of significance. The Holm correction (modified Bonferroni) will be employed on a family-wise basis to control for multiple comparisons of secondary outcomes.

Complete details of statistical analyses and methods, including data handling conventions, will be contained in a separate statistical analysis plan which will be finalized before the database is locked.

Primary efficacy analysis The primary efficacy analysis will be conducted in the full analysis set. A Pearson's Chi-squared test will be used to compare the proportions of subjects achieving clinical remission between the two treatment arms. The $95 \%$ confidence interval for the odds of achieving clinical remission at week 48 will also be constructed. Analyses will be repeated in the per protocol set.

Secondary efficacy analyses Secondary efficacy analyses will be conducted in the full analysis set. Proportions of patients achieving remission or other binary responses will be compared between groups using Pearson's Chi-squared tests. Changes in Gaussian-distributed continuous interval variables over time will be compared between groups using mixed between-within subjects ANOVA; if the data do not meet the assumptions of the test, approaches such as rank transformation will be used. Time to event analyses will be conducted using log-rank tests. Analyses will be repeated in the per protocol set.

Other planned analyses Immunological, gene expression and histological parameters will be compared between the two treatment arms using the same approaches listed for the secondary analyses with appropriate bioinformatics as indicated; subgroup analyses will repeat the between-arm comparison, split by remission status (achieved/not achieved).

Clinical, imaging, synovial and immunological factors associated with the odds of early and sustained remission will be investigated using univariable and multivariable binary logistic regression models.
RAMRIS score is considered a secondary efficacy outcome and will be analysed accordingly. For the remaining exploratory imaging and biological endpoints, descriptive summaries will be provided for all variables but inferential tests will not be performed.

Missing data For response variables (including the primary outcome) patients who discontinue study medication for lack of efficacy will be considered non-responders from that point forward. In all other instances, missing data will be addressed using multiple imputation. This technique assumes the data are missing at random; additional sensitivity analyses will include complete case analysis, best case/worst case single imputation, and imputation of a range of values to test the possible implications for the study conclusions if data are missing not at random.

\section{Ethical approval}

Ethical approval was obtained from Leeds West Research Ethics Committee (REC reference number: 10/H1307/138).

\section{Discussion}

This pragmatic, randomised phase IV, open-label study is comparing first line ETN and MTX combination therapy with a TT regimen starting with MTX monotherapy regimen with SDMARD escalation followed by ETN and MTX at week 24 if the treatment target of remission (or LDA with physician impression of remission) is not achieved.

The fundamental objective of this study is to determine whether TNFi instituted as first-line therapy in early RA confers better outcomes (including depth of remission and exploratory parameters indicating normalisation of immune system aberration) compared to a conventional TT therapeutic approach with delayed TNFi if required. The VEDERA study will contribute to the existing evidence $[37,38]$ and in addition addresses the question of whether quality of response to TNFi following MTX-failure differs from that achieved with firstline use, prior to MTX exposure. The accurate imaging aligned with the clinical assessments will also ensure correct categorisation of patient response, to address the weaknesses of clinical composite measures for assessing remission [39].

Although the planned sample size $(n=120)$ is relatively small, the study is adequately powered to detect a clinically meaningful difference in remission rate, based on existing published data. Secondary outcomes are expected to be correlated with the primary outcome and we therefore anticipate the effect size to be similar for these variables, which are considered supportive of the primary hypothesis. For exploratory endpoints the focus will be purely descriptive; the sample size has been 
inflated to help ensure that planned subgroup analyses of patients receiving delayed ETN will include sufficient patients, based on rules of thumb for pilot studies.

One of the secondary objectives of the study is remission maintenance and sustainability after ETN withdrawal. There is increasing published data regarding this [40], and while this study does not primarily seek to investigate drug tapering following remission, it will provide additional insights. Finally, the biosamples collected will allow investigation of the pathobiology of disease, mechanisms of drug action and predictors of response and remission in order to enable more effective tailoring of therapy in clinical practice.

\begin{abstract}
Abbreviations
ACPA: Anti-cyclic citrullinated peptide antibody; ACR: American college of rheumatology; CDAl: Clinical disease activity index; CV: Cardiovascular; HS-CRP: High sensitivity C-reactive protein; DAS: Disease activity score; DMARD: Disease modifying anti-rheumatic drug; sDMARD: synthetic DMARD; bDMARD: biological DMARD; DCE-MRI: Dynamic contrast enhanced magnetic resonance imaging; ESR: Erythrocyte sedimentation rate; ETN: Etanercept; EULAR: European league against rheumatism; EQ-5D: Euro quality of life five dimensions questionnaire; HAQ: Health assessment questionnaire; HCQ: Hydroxychloroquine; HRUS: High resolution ultrasonography; LDA: Low disease activity; MTX: Methotrexate; MRI: Magnetic resonance imaging; NICE: National institute of clinical excellence; NSAID: Nonsteroidal anti-inflammatory drug; OMERACT: Outcome measures in RA clinical trials; RA: Rheumatoid arthritis; RF: Rheumatoid factor; RAQoL: RA quality of life questionnaire; RA-WIS: RA work instability questionnaire; RAMRIS: RA magnetic resonance imaging score; SDAl: Simplified disease activity index; SSZ: Sulfasalazine; TNF: Tumour necrosis factor; TNFi: Tumour necrosis factor inhibitor; TT: Treat to target (current recommendations for best practice: rapid escalation of treatment to achieve a pre-defined target of disease control, with monthly disease activity assessment); VAS: Visual analogue scale.
\end{abstract}

\section{Competing interests}

MHB has received honoraria/consultancy/grant funding from Abbvie, Bristol-Myers-Squibb, Roche-Chugai, Pfizer and UCB. PE has received honoraria/ consultancy/grant funding from Abbvie, Bristol-Myers-Squibb, MSD, Roche-Chugai, Pfizer, UCB, Novartis and Lilly. No known competing interests exist with any of the remaining authors named above.

\section{Authors' contributions}

$M H B, S H, R D, R J W, E M A H, P E$ participated in the design of the study and helped to draft the manuscript. MHB conceived the study. MHB and SH led the drafting of the trial protocol and participated in the co-ordination of the study. RBD led the drafting of the protocol paper and participated in the co-ordination of the study. EMAH was responsible for the design of the statistical analysis of the study. All authors read and approved the final manuscript.

\section{Author details}

'Leeds Institute of Rheumatic and Musculoskeletal Medicine, University of Leeds, Leeds, UK. ${ }^{2} \mathrm{NIHR}$ Leeds Musculoskeletal Biomedical Research Unit, Leeds Teaching Hospitals NHS Trust, Leeds, UK. ${ }^{3}$ University of Manchester Centre for Imaging Sciences, Manchester, UK.

Received: 13 May 2015 Accepted: 2 February 2016

Published online: 05 February 2016

\section{References}

1. Tiippana-Kinnunen T, Paimela L, Peltomaa R, Kautiainen H, Laasonen L, Leirisalo-Repo M. Work disability in Finnish patients with rheumatoid arthritis: a 15-year follow-up. Clin Exp Rheumatol. 2014;32:88-94.

2. Turesson C, McClelland RL, Christianson T, Matteson E. Clustering of extraarticular manifestations in patients with rheumatoid arthritis. J Rheumatol. 2008;35:179-80.
3. Atar D, Birkeland Kl, Uhlig T. "Treat to target": moving targets from hypertension, hyperlipidaemia and diabetes to rheumatoid arthritis. Ann Rheum Dis. 2010;69:629-30.

4. Goekoop-Ruiterman YPM, de Vries-Bouwstra JK, Kerstens PJSM, Nielen MMJ, Vos $K$, van Schaardenburg D, et al. DAS-driven therapy versus routine care in patients with recent-onset active rheumatoid arthritis. Ann Rheum Dis. 2010;69:65-9.

5. Smolen JS, Aletaha D, Bijlsma JWJ, Breedveld FC, Boumpas D, Burmester G, et al. Treating rheumatoid arthritis to target: recommendations of an international task force. Ann Rheum Dis. 2010;69:631-7.

6. Grigor C, Capell H, Stirling A, McMahon AD, Lock P, Vallance R, et al. Effect of a treatment strategy of tight control for rheumatoid arthritis (the TICORA study): a single-blind randomised controlled trial. Lancet. 2004;364:263-9.

7. Landewé RBM, Boers M, Verhoeven AC, Westhovens R, van de Laar MAFJ, Markusse HM, et al. COBRA combination therapy in patients with early rheumatoid arthritis: long-term structural benefits of a brief intervention. Arthritis Rheum. 2002:46:347-56.

8. Saunders SA, Capell HA, Stirling A, Vallance R, Kincaid W, McMahon AD, et al. Triple therapy in early active rheumatoid arthritis: a randomized, single-blind, controlled trial comparing step-up and parallel treatment strategies. Arthritis Rheum. 2008;58:1310-7.

9. Weinblatt ME, Keystone EC, Furst DE, Moreland LW, Weisman MH, Birbara CA, et al. Adalimumab, a fully human anti-tumor necrosis factor alpha monoclonal antibody, for the treatment of rheumatoid arthritis in patients taking concomitant methotrexate: the ARMADA trial. Arthritis Rheum. 2003;48:35-45.

10. Maini R, St Clair EW, Breedveld F, Furst D, Kalden J, Weisman M, et al. Infliximab (chimeric anti-tumour necrosis factor alpha monoclonal antibody) versus placebo in rheumatoid arthritis patients receiving concomitant methotrexate: a randomised phase III trial. ATTRACT Study Group. Lancet. 1999;354:1932-9.

11. Moreland LW, Baumgartner SW, Schiff MH, Tindall EA, Fleischmann RM, Weaver AL, et al. Treatment of rheumatoid arthritis with a recombinant human tumor necrosis factor receptor (p75)-Fc fusion protein. N Engl J Med. 1997;337:141-7.

12. Klareskog L, van der Heijde D, de Jager JP, Gough A, Kalden J, Malaise M, et al. Therapeutic effect of the combination of etanercept and methotrexate compared with each treatment alone in patients with rheumatoid arthritis: double-blind randomised controlled trial. Lancet. 2004;363:675-81.

13. Van Riel PLCM, Taggart AJ, Sany J, Gaubitz M, Nab HW, Pedersen R, et al. Efficacy and safety of combination etanercept and methotrexate versus etanercept alone in patients with rheumatoid arthritis with an inadequate response to methotrexate: the ADORE study. Ann Rheum Dis. 2006;65:1478-83.

14. Smolen JS, Landewé R, Breedveld FC, Buch M, Burmester G, Dougados M, et al. EULAR recommendations for the management of rheumatoid arthritis with synthetic and biological disease-modifying antirheumatic drugs: 2013 update. Ann Rheum Dis. 2014;73:492-509.

15. National Collaborating Centre for Chronic Conditions. The management of rheumatoid arthritis in adults. 2009.

16. Emery P, Breedveld FC, Hall S, Durez P, Chang DJ, Robertson D, et al. Comparison of methotrexate monotherapy with a combination of methotrexate and etanercept in active, early, moderate to severe rheumatoid arthritis (COMET): a randomised, double-blind, parallel treatment trial. Lancet. 2008;372:375-82.

17. Smolen JS, Van Der Heijde DMFM, St Clair EW, Emery P, Bathon JM, Keystone E, et al. Predictors of joint damage in patients with early rheumatoid arthritis treated with high-dose methotrexate with or without concomitant infliximab: results from the ASPIRE trial. Arthritis Rheum. 2006;54:702-10.

18. Smolen JS, Emery P, Fleischmann R, van Vollenhoven RF, Pavelka K, Durez P, et al. Adjustment of therapy in rheumatoid arthritis on the basis of achievement of stable low disease activity with adalimumab plus methotrexate or methotrexate alone: the randomised controlled OPTIMA trial. Lancet. 2014;383:321-32.

19. Emery P, Kvien TK, Combe B, Freundlich B, Robertson D, Ferdousi $T$, et al. Combination etanercept and methotrexate provides better disease control in very early ( $<=4$ months) versus early rheumatoid arthritis ( $>4$ months and $<2$ years): post hoc analyses from the COMET study. Ann Rheum Dis. 2012;71:989-92.

20. Quinn MA, Conaghan PG, O'Connor PJ, Karim Z, Greenstein A, Brown A, et al. Very early treatment with infliximab in addition to methotrexate in 
early, poor-prognosis rheumatoid arthritis reduces magnetic resonance imaging evidence of synovitis and damage, with sustained benefit after infliximab withdrawal: results from a twelve-month randomized, doubleblind, placebo-controlled trial. Arthritis Rheum. 2005:52:27-35.

21. Quinn MA, Emery P. Window of opportunity in early rheumatoid arthritis: possibility of altering the disease process with early intervention. Clin Exp Rheumatol. 2003;21(5 Suppl 31):S154-7.

22. Nam JL, Villeneuve $\mathrm{E}$, Hensor EMA, Conaghan PG, Keen HI, Buch MH, et al. Remission induction comparing infliximab and high-dose intravenous steroid, followed by treat-to-target: a double-blind, randomised, controlled trial in new-onset, treatment-naive, rheumatoid arthritis (the IDEA study). Ann Rheum Dis. 2014;73:75-85.

23. Emery P, Hammoudeh M, FitzGerald O, Combe B, Martin-Mola E, Buch MH, et al. Sustained Remission with Etanercept Tapering in Early Rheumatoid Arthritis. N Engl J Med. 2014;371:1781-92.

24. Ton E, Bakker MF, Verstappen SMM, Ter Borg EJ, van Albada-Kuipers IA, Schenk Y, et al. Look beyond the disease activity score of 28 joints (DAS28): tender points influence the DAS28 in patients with rheumatoid arthritis. J Rheumatol. 2012;39:22-7.

25. Saleem B, Brown AK, Keen H, Nizam S, Freeston J, Wakefield R, et al. Should imaging be a component of rheumatoid arthritis remission criteria? A comparison between traditional and modified composite remission scores and imaging assessments. Ann Rheum Dis. 2011;70:792-8.

26. Van der Heijde DM, van't Hof M, van Riel PL, van de Putte LB. Development of a disease activity score based on judgment in clinical practice by rheumatologists. J Rheumatol. 1993;20:579-81.

27. Aletaha D, Nell VPK, Stamm T, Uffmann M, Pflugbeil S, Machold K, et al. Acute phase reactants add little to composite disease activity indices for rheumatoid arthritis: validation of a clinical activity score. Arthritis Res Ther. 2005;7:R796-806.

28. Smolen JS, Breedveld FC, Schiff MH, Kalden JR, Emery P, Eberl G, et al. A simplified disease activity index for rheumatoid arthritis for use in clinical practice. Rheumatol Oxf Engl. 2003;42:244-57.

29. van Gestel AM, Prevoo ML, Hof MA v't, van Rijswijk MH, van de Putte LB, van Riel PL. Development and validation of the European League Against Rheumatism response criteria for rheumatoid arthritis. Comparison with the preliminary American College of Rheumatology and the World Health Organization/International League Against Rheumatism Criteria. Arthritis Rheum. 1996;39:34-40.

30. Felson DT, Anderson JJ, Boers M, Bombardier C, Furst D, Goldsmith C, et al. American College of Rheumatology. Preliminary definition of improvement in rheumatoid arthritis. Arthritis Rheum. 1995;38:727-35.

31. Fries JF, Spitz PW, Young DY. The dimensions of health outcomes: the health assessment questionnaire, disability and pain scales. J Rheumatol. 1982;9:789-93.

32. De Jong $Z$, van der Heijde D, McKenna SP, Whalley D. The reliability and construct validity of the RAQoL: a rheumatoid arthritis-specific quality of life instrument. Br J Rheumatol. 1997;36:878-83.

33. Group EQ. EuroQol-a new facility for the measurement of health-related quality of life. Health Policy Amst Neth. 1990;16:199-208.

34. Gilworth G, Chamberlain MA, Harvey A, Woodhouse A, Smith J, Smyth MG, et al. Development of a work instability scale for rheumatoid arthritis. Arthritis Rheum. 2003;49:349-54.

35. Østergaard M, Peterfy C, Conaghan P, McQueen F, Bird P, Ejbjerg B, et al. OMERACT Rheumatoid Arthritis Magnetic Resonance Imaging Studies. Core set of MRI acquisitions, joint pathology definitions, and the OMERACT RAMRI scoring system. J Rheumatol. 2003;30:1385-6.

36. Genant HK, Jiang Y, Peterfy C, Lu Y, Redei J, Countryman PJ. Assessment of rheumatoid arthritis using a modified scoring method on digitized and original radiographs. Arthritis Rheum. 1998;41:1583-90.

37. Moreland LW, O'Dell JR, Paulus HE, Curtis JR, Bathon JM, St. Clair EW, et al. A randomized comparative effectiveness study of oral triple therapy versus etanercept plus methotrexate in early aggressive rheumatoid arthritis: The Treatment of Early Aggressive Rheumatoid Arthritis trial. Arthritis Rheum. 2012;64:2824-35

38. Takeuchi T, Yamanaka H, Ishiguro N, Miyasaka N, Mukai M, Matsubara T, et al. Adalimumab, a human anti-TNF monoclonal antibody, outcome study for the prevention of joint damage in Japanese patients with early rheumatoid arthritis: the HOPEFUL 1 study. Ann Rheum Dis. 2014;73:536-43.

39. Brown AK, Conaghan PG, Karim Z, Quinn MA, Ikeda K, Peterfy CG, et al. An explanation for the apparent dissociation between clinical remission and continued structural deterioration in rheumatoid arthritis. Arthritis Rheum. 2008:58:2958-67.

40. Smolen JS, Nash P, Durez P, Hall S, llivanova E, Irazoque-Palazuelos F, et al.: Maintenance, reduction, or withdrawal of etanercept after treatment with etanercept and methotrexate in patients with moderate rheumatoid arthritis (PRESERVE): a randomised controlled trial. The Lancet 16, 381:918-29

\section{Submit your next manuscript to BioMed Central and we will help you at every step:}

- We accept pre-submission inquiries

- Our selector tool helps you to find the most relevant journal

- We provide round the clock customer support

- Convenient online submission

- Thorough peer review

- Inclusion in PubMed and all major indexing services

- Maximum visibility for your research

Submit your manuscript at www.biomedcentral.com/submit
O BioMed Central 